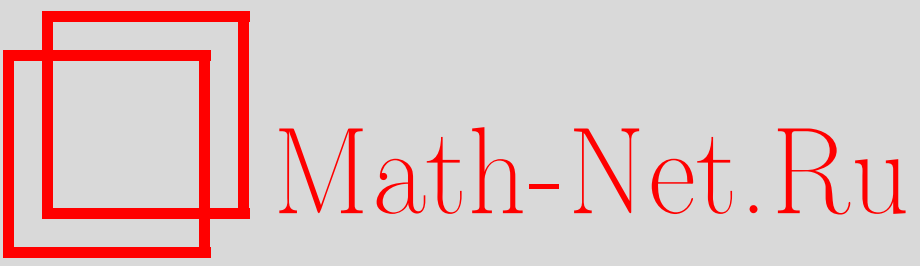

А. А. Власов, Проблема радиационной отдачи для классических заряженных пылинок, ТМФ, 2003, том 134, номер 2, 254-272

DOI: https://doi.org/10.4213/tmf156

Использование Общероссийского математического портала Math-Net.Ru подразумевает, что вы прочитали и согласны с пользовательским соглашением

http://www.mathnet.ru/rus/agreement

Параметры загрузки:

IP : 54.80 .97 .219

26 апреля 2023 г., 16:49:54 
ТЕОРЕТИЧЕСКАЯ

И МАТЕМАТИЧЕСКАЯ

ФИЗИКА

Том 134, № 2

февраль, 2003

(C) 2003 г.

А. А. Власов*

\section{ПРОБЛЕМА РАДИАЦИОННОЙ ОТДАЧИ ДЛЯ КЛАССИЧЕСКИХ ЗАРЯЖЕННЫХ ПЫЛИНОК}

Для модели Зоммерфельда протяженной заряженной частицы ("пылинки") разобраны некоторые классические задачи (кулоновское рассеяние, движение в постоянных внешних электромагнитных полях, броуновское движение). При этом выявлены существенные отличия результатов от классических как в количественном отношении (изменение угла при кулоновском рассеянии, уменьшение коэффициента диффузии для броуновской частицы, замедление раскрутки "пылинки" в циклотроне), так и в качественном (возникновение эффекта туннелирования через потенциальный барьер). При стремлении размера частицы к нулю указанные отличия не исчезают. Следовательно, уравнение движения протяженного объекта не имеет гладкого предельного перехода $\mathrm{K}$ ньютоновскому уравнению для точки.

Ключевые слова: классическая электродинамика, реакция излучения.

\section{1. ВВЕДЕНИЕ}

Из классической электродинамики известно, что движущееся с ускорением заряженное тело должно излучать электромагнитные волны и, следовательно, испытывать обратную отдачу излучения. Как учесть такую отдачу в классических уравнениях движения тела?

Проблеме учета радиационной отдачи вот уже более ста лет (если их отсчитывать от трудов Лоренца и Абрагама [1]), однако до сих пор она дискутируется в литературе и выходят статьи, ей посвященные (например, по квантовым аспектам проблемы см. работы [2], по классическим - [3]-[6]).

Проблема ставится следующим образом. Если в рамках некоторой классической задачи можно пренебречь размерами излучающего тела (по сравнению с другими характерными размерами в данной задаче), то возникает желание использовать понятие “точечности” для описания такого тела и ввести для этого дельта-функцию. Однако введение дельта-функции приводит к появлению расходимостей в классическом подходе и, следовательно, к необходимости перенормировки массы частицы. Такую процедуру в релятивистском случае в рамках классической электродинамики впервые выполнил Дирак [7] и получил свое знаменитое уравнение (в литературе оно известно как уравнение Лоренца-Дирака), учитывающее обратную реакцию излучения на движение точечного релятивистского классического тела.

\footnotetext{
* Московский государственный университет, Москва, Россия. E-mail: vlasov@srdlan.npi.msu.su
} 
Однако тут же было установлено (в том числе и самим Дираком), что такое уравнение, если его рассматривать как точное, приводит ко многим парадоксам. Среди них в литературе (см., например, [8]-[15], [5]) обычно отмечаются следующие:

а) саморазгон (в отсутствие внешних сил точечное тело начинает самопроизвольно разгоняться до скорости света);

б) предускорение (если наложить дополнительное условие и исключить саморазгоняюшееся решение, то остается решение, которое описывает точечное тело, "чувствующее” действие силы заранее, с опережением по времени);

в) наличие "экзотических" (нетрадиционных) решений в задаче о лобовом столкновении (например, при одномерном движении двух разноименных точечных зарядов возникают решения, описываюшие их взаимное отталкивание).

Можно привести и другие примеры.

Поэтому возникает необходимость модификации (а то, что это надо делать - мнение многих современных физиков, см., например, [2], [3]) уравнения Лоренца-Дирака. Здесь возможны два различных подхода:

а) отнести проблему “точечности” к области квантовой теории и рассматривать модели взаимодействия заряженных элементарных частиц и их полей в рамках квантовых представлений;

б) остаться в рамках классической теории (считая вслед за Дираком [7], что проблема радиационной отдачи не требует привлечения квантового подхода), но отказаться от “точечности” и рассматривать классические объекты малой протяженности (например, заряженные пылинки космической пыли, пылевую плазму, броуновские частицы с зарядом и т.п.).

В данной работе автор будет придерживаться второго подхода, т.е. рассматривать уравнения движения классического протяженного тела с учетом радиационной отдачи.

Одной из простейших моделей, учитывающей конечную протяженность заряженного тела, является модель Зоммерфельда жесткой сферы радиуса $a$, массы $m$ и заряда $Q$ (частица Зоммерфельда) [16]. В так называемом квазистационарном нерелятивистском приближении [13]-[15], когда понятие жесткого закрепления зарядов вполне применимо и может реализовываться, например, на диэлектрической пылинке, уравнение движения протяженной частицы Зоммерфельда принимает вид

$$
m \dot{\vec{v}}=\vec{F}_{\text {ext }}+\eta\left[\vec{v}\left(t-\frac{2 a}{c}\right)-\vec{v}(t)\right] .
$$

Здесь $a$ - радиус сферы, $\eta=Q^{2} /\left(3 c a^{2}\right), \vec{v}=d \vec{R} / d t, \vec{R}$ - координата центра сферы, $\vec{F}_{\text {ext }}$ - внешняя сила.

Решения уравнения (1) можно искать одним из аналитических методов, разработанных для дифференциально-разностных уравнений. Однако в некоторых случаях бывает удобным численное интегрирование.

\section{2. ТУННЕЛИРОВАНИЕ}

Уравнение (1) имеет решения, которые можно интерпретировать в рамках эффекта классического туннелирования: благодаря запаздыванию частица отвечает на действие потенциальной силы слишком поздно и проскакивает барьер (эффект туннелирова- 
ния для точечного тела, подчиняющегося уравнению Лоренца-Дирака, установлен в работе [4]).

Рассмотрим нерелятивистское решение уравнения (1) для потенциального барьера, образованного однородным статическим электрическим полем $E_{z}$, направленным вдоль оси $z$ и отличным от нуля в слое $0<z<S$ (модель плоского конденсатора),

$$
E_{z}= \begin{cases}0, & z<0 \\ E, & 0<z<S, \\ 0, & S<z .\end{cases}
$$

Предположим, что частица движется вдоль оси $z$, т.е. $\vec{R}=(0,0, R)$. Введем безразмерные переменные $y=R / L, \tau=c t / L, \delta=2 a / L$ и положим для простоты $\delta=1$ и $S=L=2 a$.

Внешняя сила, определяемая полем $E_{z}$, есть

$$
F_{\text {ext }}=\int d \vec{r} \rho E_{z}=E Q f
$$

где плотность заряда сферы Зоммерфельда дается фоормулой

$$
\rho=\frac{Q \delta(|\vec{r}-\vec{R}|-a)}{4 \pi a^{2}} .
$$

Функция $f$ в формуле (2) имеет вид

$$
f= \begin{cases}0, & y<-\frac{1}{2} \\ \frac{2 y+1}{2}, & -\frac{1}{2}<y<\frac{1}{2}, \\ \frac{-2 y+3}{2}, & \frac{1}{2}<y<\frac{3}{2} \\ 0, & \frac{3}{2}<y .\end{cases}
$$

Она возникает вследствие конечности размера частицы и ограниченности области пространства, в котором сушествует электрическое поле. Действительно, сила $F_{\text {ext }}$ для частицы Зоммерфельда в плоском конденсаторе записывается как

$$
F_{\text {ext }}=\int d \vec{r} \rho E_{z}=\frac{Q E}{4 \pi a^{2}} \int d \vec{r} \delta(|\vec{r}-\vec{R}|-a)[\theta(z)-\theta(z-S)],
$$

где $\theta(z)$ - функция Хевисайда. Введем новую переменную $\vec{\xi}, \vec{r} \equiv \vec{\xi}+\vec{R}, d \vec{r}=d \vec{\xi}=$ $\xi^{2} d \xi \sin \vartheta d \vartheta d \phi$. Полагая $\cos \vartheta \equiv \mu$, получаем $z=R+\xi \mu$. Интегрируя по углу $\phi$, имеем

$$
\begin{aligned}
F_{\text {ext }} & =\frac{Q E}{2 a} \int_{-1}^{+1} d \mu[\theta(R+a \mu)-\theta(R+a \mu-S)]= \\
& =\frac{Q E}{2 a}\left[\int_{R-a}^{R+a} d w \theta(w)-\int_{R-a-S}^{R+a-S} d w \theta(w)\right] .
\end{aligned}
$$

Выражение в квадратных скобках легко вычисляется и при сделанном выше выборе $S=$ $L=2 a$ дает формулу (2). 
С учетом (2) уравнение движения (1) частишы Зоммерфельда принимает вид

$$
\frac{d^{2} y}{d \tau^{2}}=k\left[\frac{d y(\tau-1)}{d \tau}-\frac{d y(\tau)}{d \tau}\right]+\lambda f
$$

где $k=2 Q^{2} /\left(3 m c^{2} a\right), \lambda=L Q E /\left(m c^{2}\right)$.

Теперь рассмотрим аналогичную задачу для классической заряженной точечной частищы без излучения. Такая частища подчиняется уравнению

$$
\frac{d^{2} y}{d \tau^{2}}=F_{E}
$$

где

$$
F_{E}= \begin{cases}0, & y<0 \\ \lambda, & 0<y<1 \\ 0, & 1<y\end{cases}
$$

Согласно формуле (4) выражение для скорости на выходе из барьера, т.е. при $y=1$, имеет вид

$$
v^{2}=2 \lambda+v_{0}^{2}
$$

где $v=d y / d \tau, v_{0}$ - начальная скорость. Соответственно нулевая скорость на границе барьера $y=1$ достигается при $v_{0}=\sqrt{2|\lambda|}$. Следовательно, для начальных скоростей меньше критической,

$$
v_{0}^{2}<v_{\mathrm{cr}}^{2}, \quad v_{\mathrm{cr}}^{2}=2|\lambda|
$$

существует точка поворота - т.е. классическая частица не может преодолеть потенциальный барьер.

Решение задачи о барьере для частицы Зоммерфельда сушественно отличается от классического. Оно в обшем виде аналитически просто, однако его исследование ввиду громоздкости формул затруднено. Поэтому мы воспользовались численным расчетом. Результаты показаны на рис. 1-3 (по вертикальной оси отложена скорость $d y / d \tau$, по горизонтальной - координата $y,-0.5<y<1.5)$.

На рис. 1 взяты параметры $k=1, \lambda=-0.5, v_{\mathrm{cr}}=1.0$ (т.е. частиша Зоммерфельда массы $m$ и заряда $Q$ имеет размер $2 r_{\mathrm{cl}} / 3\left(r_{\mathrm{cl}} \equiv Q^{2} /\left(m c^{2}\right)\right)$, ширина барьера $2 a=4 r_{\mathrm{cl}} / 3$, а электрическое поле барьера $E$ по порядку величины совпадает с $\left.E_{\mathrm{cl}} \equiv Q /\left(r_{\mathrm{cl}}\right)^{2}\right)$.

Из рис. 1 следует, что при "начальных скоростях" сферы (в силу запаздывания их надо задавать не в начальной точке, а на начальном интервале) $v_{0}=0.4,0.6,0.7$ происходит поворот траектории; однако частица с начальной скоростью $v_{0}=0.8$ проходит барьер - возникает эффект туннелирования.

На рис. 2 показано возникновение эффекта туннелирования при значениях параметров $k=1, \lambda=-0.1, v_{\text {cr }}=0.44720$. Если начальные скорости сферы $v_{0}=0.12, v_{0}=$ 0.3 , то происходит поворот траектории; однако частица с начальной скоростью $v_{0}=0.4$ проходит барьер - возникает эффект туннелирования.

Сравнение кривых на рис. $3(k=10)$ с кривыми на рис. $2(k=1)$ (начальные скорости те же) показывает, что чем больше значение $k$, тем при меньших начальных скоростях возникает эффект туннелирования.

4 Теоретическая и математическая физика, т. 134, № 2, 2003 г. 


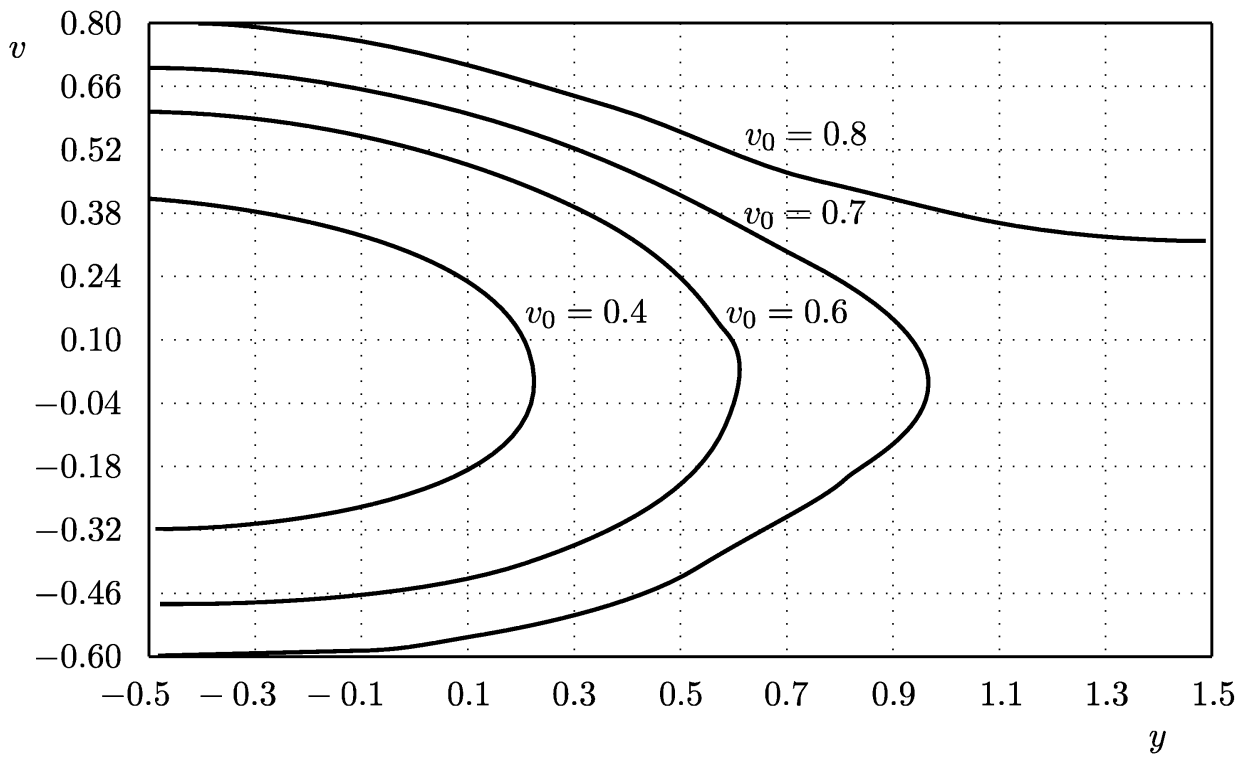

Рис. 1. Эффект туннелирования для частицы Зоммерфельда при $k=1, \lambda=-0.5, v_{0}<$ $v_{\text {cr }}=1$

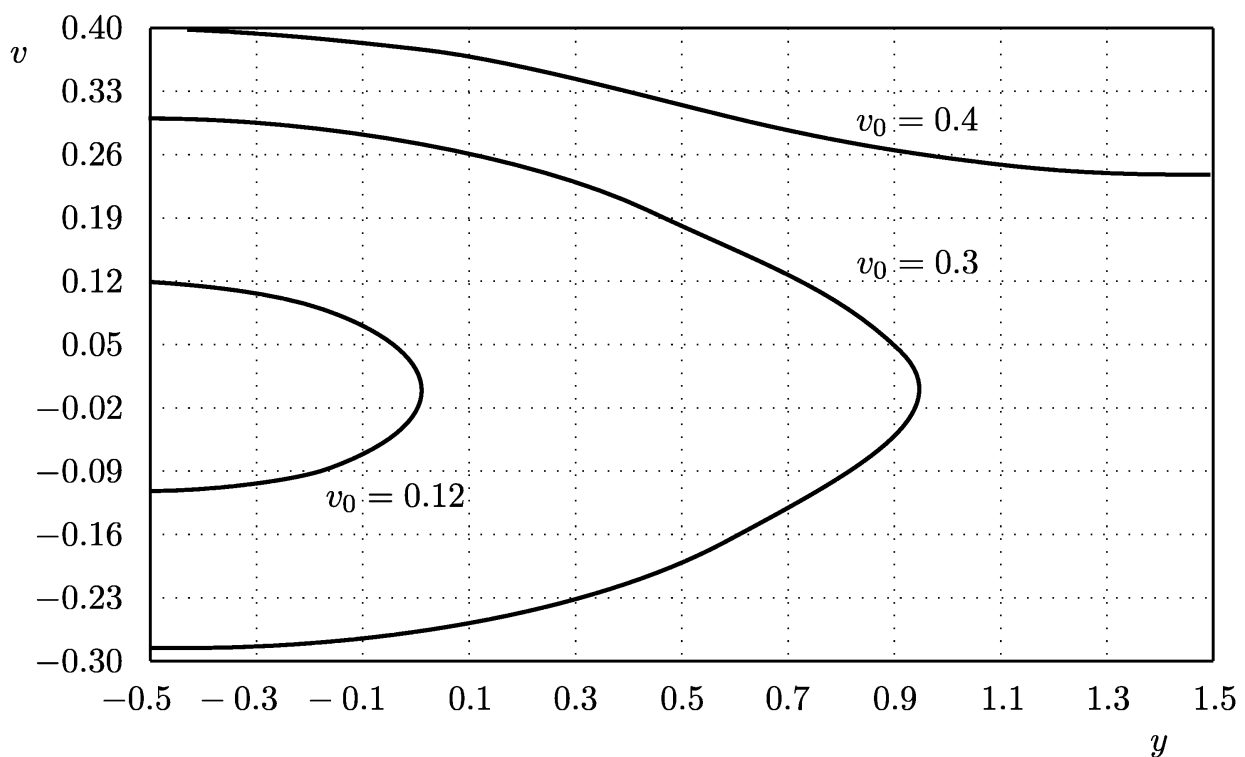

Рис. 2. Эфффект туннелирования для частицы Зоммерфельда при $k=1, \lambda=-0.1, v_{0}<$ $v_{\text {cr }}=0.4472$.

\section{3. ЧАСТИЦА В МАГНИТНОМ ПОЛЕ, ЦИКЛОТРОН}

Пусть частица Зоммерфельда радиуса $а$ движется во внешнем статическом магнитном поле $\vec{H}$, тогда внешняя сила записывается как

$$
\vec{F}_{\mathrm{ext}}=\frac{1}{c} \int d \vec{r} \rho[\dot{\vec{R}}, \vec{H}]
$$




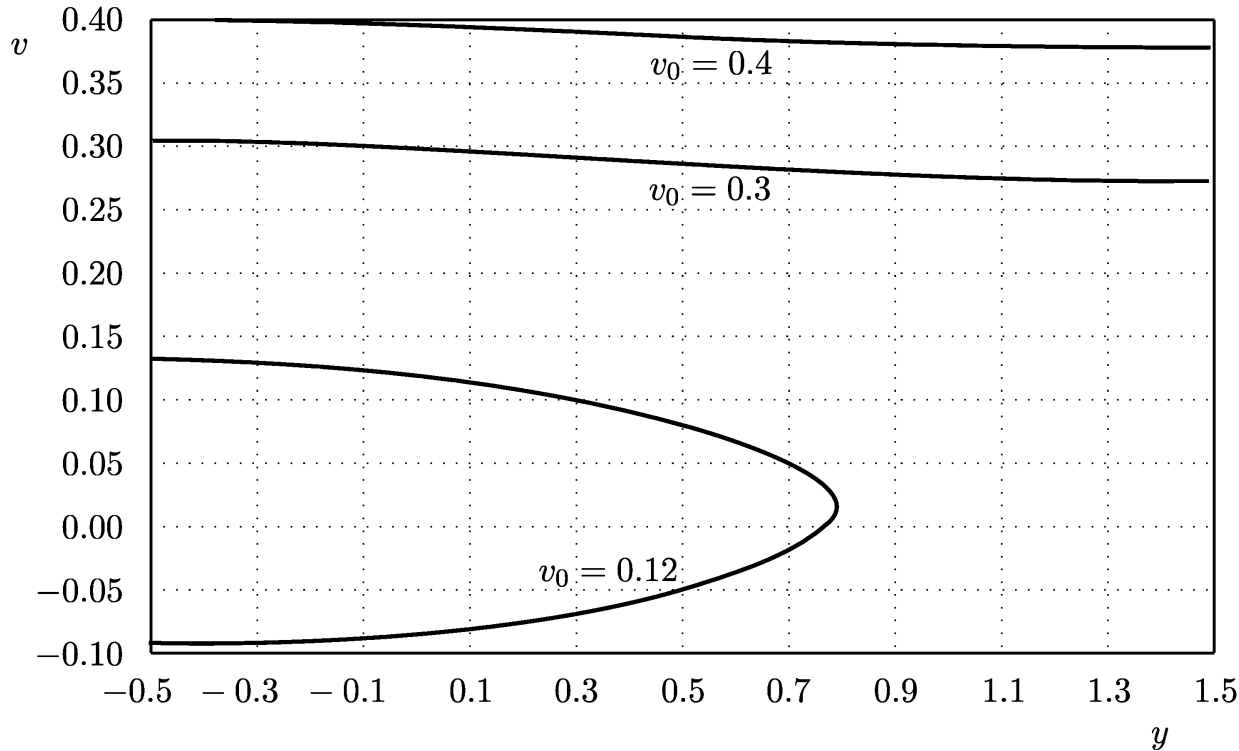

Рис. 3. Эффект туннелирования для частицы Зоммерфельда при $k=10, \lambda=-0.1, v_{0}<$ $v_{\mathrm{cr}}=0.4472$

и для $\rho=Q \delta(|\vec{r}-\vec{R}|-a) / 4 \pi a^{2}$ принимает вид

$$
\vec{F}_{\mathrm{ext}}=\frac{Q}{c}[\dot{\vec{R}}, \vec{H}]
$$

Если магнитное поле сосредоточено в слое конечной толшины $S(0<Y<S)$ и параллельно оси $z(\vec{H}=(0,0, H), \vec{R}=(X, Y, 0))$, то из-за конечности размера частицы силу $\vec{F}_{\text {ext }}(5)$ следует умножить на ступенчатую функцию

$$
f= \begin{cases}0, & Y<-a, \\ \frac{Y}{2 a}+\frac{1}{2}, & -a<Y<a, \\ 1, & a<Y<S-a, \\ \frac{S-Y}{2 a}+\frac{1}{2}, & S-a<Y<S+a, \\ 0, & S+a<Y .\end{cases}
$$

В безразмерных переменных $x=X / L, y=Y / L, \tau=c t / L$ ( $L$-масштабный множитель) уравнение (1) с учетом соотношений (5), (6) принимает вид

$$
\begin{aligned}
& \ddot{y}=k[\dot{y}(\tau-\delta)-\dot{y}(\tau)]-\lambda \dot{x} f, \\
& \ddot{x}=k[\dot{x}(\tau-\delta)-\dot{x}(\tau)]+\lambda \dot{y} f,
\end{aligned}
$$


где

$$
\begin{aligned}
& f= \begin{cases}0, & y<-\frac{\delta}{2}, \\
\frac{y}{\delta}+\frac{1}{2}, & -\frac{\delta}{2}<y<\frac{\delta}{2}, \\
1, & \frac{\delta}{2}<y<s-\frac{\delta}{2}, \\
\frac{s-y}{\delta}+\frac{1}{2}, & s-\frac{\delta}{2}<y<s+\frac{\delta}{2}, \\
0, & s+\frac{\delta}{2}<y,\end{cases} \\
& k=\frac{Q^{2} L}{3 a^{2} m c^{2}}, \quad \lambda=\frac{Q H L}{m c^{2}}, \quad \delta=\frac{2 a}{L}, \quad s=\frac{S}{L} .
\end{aligned}
$$

Классическим аналогом уравнений (7), (8) для точечной частицы без радиационного затухания является система уравнений, получаемая при $k \equiv 0$ в (7) и $\delta \equiv 0$ в (8),

$$
\begin{aligned}
& \ddot{y}=-\lambda \dot{x} g, \\
& \ddot{x}=\lambda \dot{y} g,
\end{aligned}
$$

где

$$
g= \begin{cases}0, & y<0 \\ 1, & 0<y<s, \\ 0, & s<y .\end{cases}
$$

Выбирая начальные условия $x(0)=0, y(0)=0, \dot{x}(0)=0, \dot{y}(0)=v$, имеем следуюшее решение системы (9):

$$
\begin{aligned}
& x=-\frac{v}{\lambda}+\frac{v}{\lambda} \cos (\lambda \tau), \\
& y=\frac{v}{\lambda} \sin (\lambda \tau), \quad 0<y<s .
\end{aligned}
$$

Отсюда следует, что если начальные скорости меньше критической $v_{\mathrm{cr}}=\lambda s$, то траектория частицы (полуокружность) целиком лежит внутри магнитного слоя и частица не может перейти через "потенциальный барьер" и войти в "запрешенную" область $y>s$. Для $s=10^{4}$ и $\lambda=10^{-4}$ величина критической скорости $v_{\mathrm{cr}}=1$.

Уравнения (7) численно проинтегрированы для следующих значений скорости, толшины слоя и параметров частишы Зоммерфельда:

$$
\begin{aligned}
v & =0.43, \quad v=0.44, \\
s=10^{4}, \quad \lambda & =10^{-4}, \quad \delta=1.0, \quad k=\frac{4}{3 \delta^{2}},
\end{aligned}
$$

т.е. частица выбрана с зарядом и массой порядка электронной, магнитное поле $H \approx$ $10^{12} \Gamma$ с, толшина слоя $S \approx 5.0 \cdot 10^{-9}$ см (скорости в силу запаздывания выбираются на начальном интервале).

Результат интегрирования представлен на рис. 4 , где он сравнивается с классической траекторией (11) при $v=0.44$ (по горизонтальной оси отложена координата частицы $x$, по вертикальной $-y$ ). Мы отчетливо видим возникновение эффекта туннелирования для частишы Зоммерфельда: при скорости $v=0.43$ частиша движется в разрешенной области $y<s$, но при $v=0.44$, которая меньше критической $\left(v_{\mathrm{cr}}=1\right)$, частица проходит барьер и попадает в “запрешенную” область $y>s=10^{4}$. 


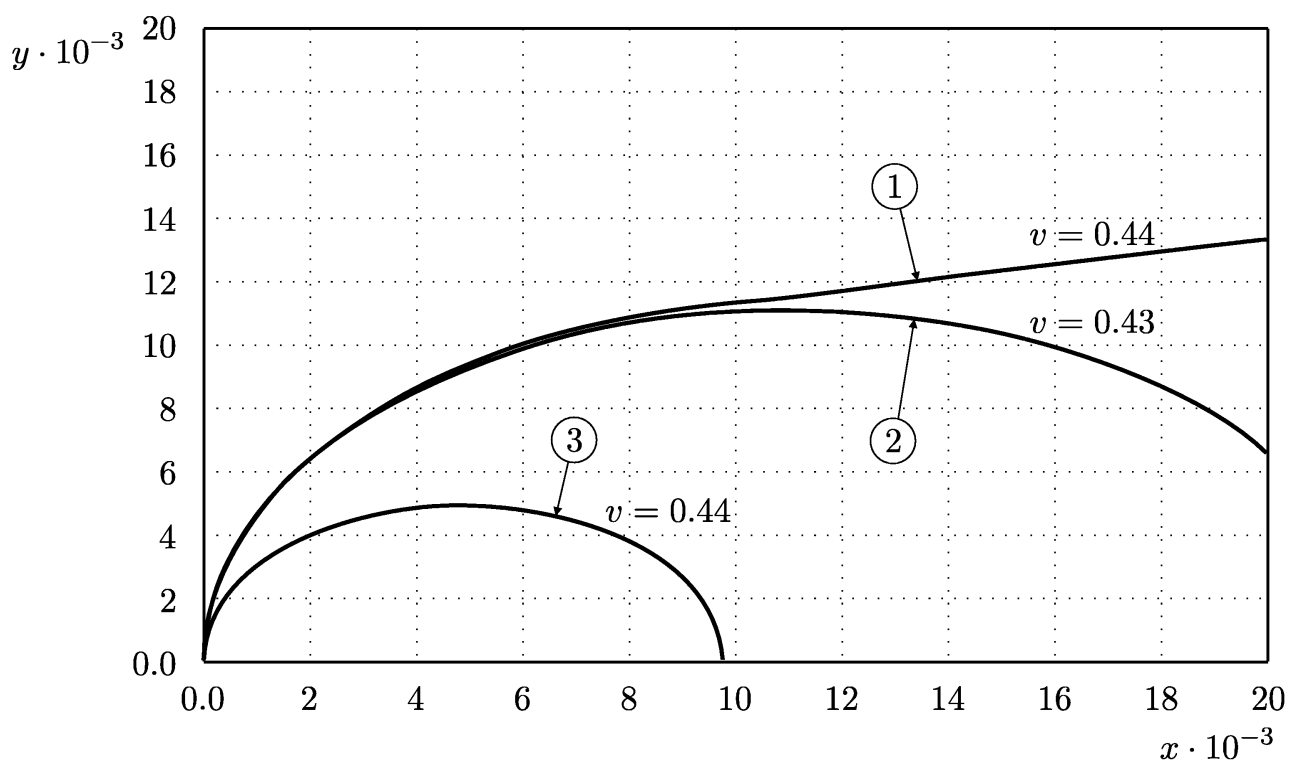

Рис. 4. Траектории частицы Зоммерфельда в магнитном поле (кривые 1,2 ) и классическая траектория (кривая 3 ).

Теперь рассмотрим движение частицы Зоммерфельда в скрещенных полях. Пусть статическое магнитное поле параллельно оси $z$ для $y<0$ и $y>s$ и обрашается в ноль при $0<y<s$. Пусть для $0<y<s$ существует такое статическое электрическое поле $E$, параллельное оси $y$, что оно всегда коллинеарно $y$-компоненте скорости частицы (частица всегда ускоряется электрическим полем в зазоре $0<y<s$ ). При этих предположениях получаем простейшую модель циклотрона. Уравнения движения частицы Зоммерфельда в циклотроне имеют вид

$$
\begin{aligned}
& \ddot{y}=k[\dot{y}(\tau-\delta)-\dot{y}(\tau)]-\lambda \dot{x} f+\epsilon \operatorname{sgn}(\dot{y})(1-f), \\
& \ddot{x}=k[\dot{x}(\tau-\delta)-\dot{x}(\tau)]+\lambda \dot{y} f,
\end{aligned}
$$

где

$$
\epsilon=\frac{Q E L}{m c^{2}}
$$

Классический аналог системы (12) получается при $k=0$ и замене функции $f$ на $g(10)$,

$$
\begin{aligned}
& \ddot{y}=-\lambda \dot{x} g+\epsilon \operatorname{sgn}(\dot{y})(1-g), \\
& \ddot{x}=\lambda \dot{y} g .
\end{aligned}
$$

Начальные условия выберем в виде $x(0)=y(0)=\dot{x}(0)=\dot{y}(0)=0$.

Согласно классическому уравнению движения без радиационного трения (13) частиша движется по раскручиваюшейся спирали. Полный прирост кинетической энергии частицы $\dot{x}^{2} / 2+\dot{y}^{2} / 2$ есть $W_{\mathrm{c}}=N \epsilon s$, где $N$ - число прохождений частицы зазора с ускоряюшим электрическим полем $E$. Если $N=10, \epsilon=\lambda=10^{-7}, s=10^{5}$, то $W_{\mathrm{c}}=10^{-1}$. 
Уравнения движения частицы Зоммерфельда (12) были численно проинтегрированы с нулевыми начальными условиями (скорости для частищы Зоммерфельда выбираются нулевыми на начальном интервале $-\delta<\tau<0)$ и значениями параметров

$$
s=10^{5}, \quad \lambda=10^{-7}=\epsilon, \quad \delta=0.3, \quad k=2.0,
$$

т.е. частища имеет массу и заряд порядка массы и заряда электрона, магнитное поле $H \approx 8.0 \cdot 10^{7} \Gamma$, электрическое поле в зазоре соответствует разности потенциалов (в энергетических единицах) $10^{4}$ эВ, величина зазора приближенно равна $10^{-7} \mathrm{~cm}$.

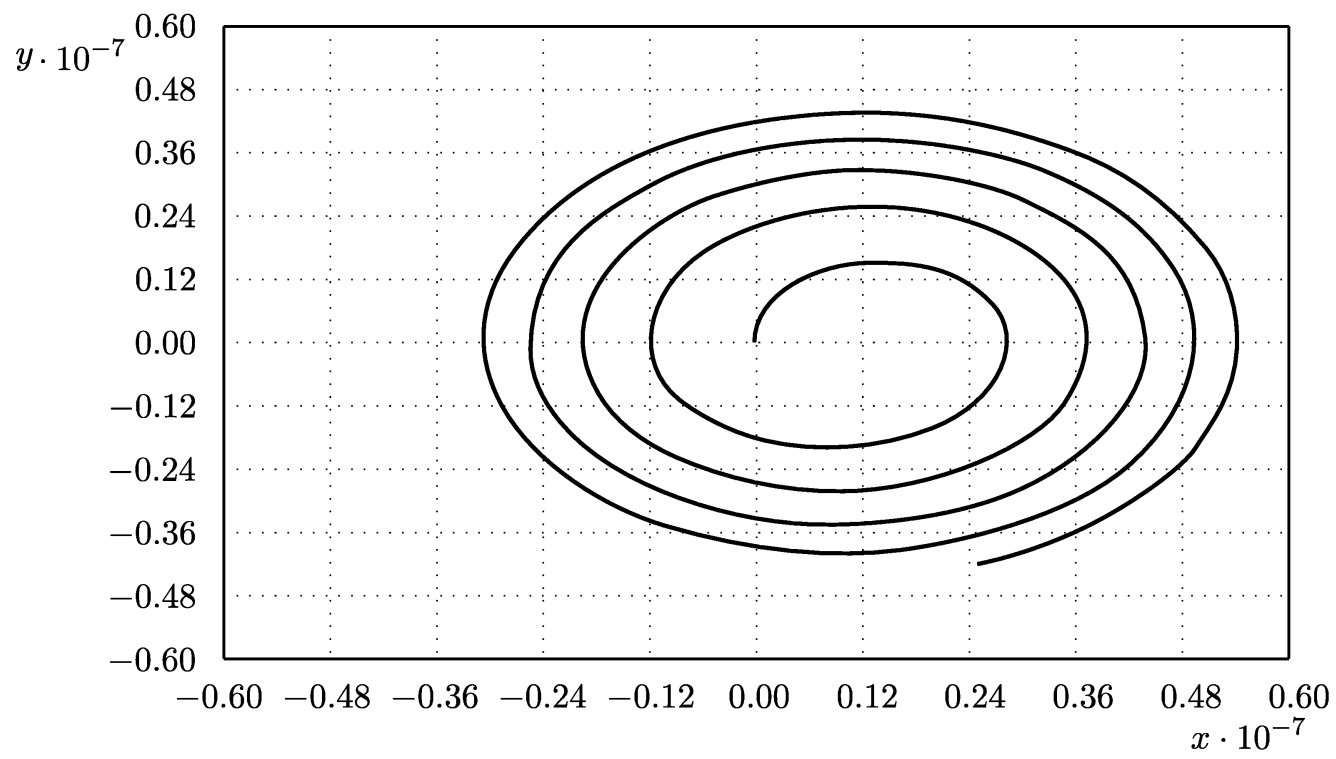

Рис. 5. Траектории классической частицы в циклотроне.

Результаты вычислений представлены на рис. 5 для классического случая и на рис. 6 для частицы Зоммерфельда (по горизонтальной оси отложено $x \lambda$, по вертикальной $y \lambda$ ). Мы видим, что для одного и того же "времени" $\tau \approx 10^{8}$ (т.е. $t \approx 10^{-4}$ c) классическая частица без учета радиационного трения совершит $N=10$ проходов через зазор с полным приростом кинетической энергии $W_{\text {clas }}=0.1$, тогда как частица Зоммерфельда пройдет зазор только $N=6$ раз с полным приростом кинетической энергии $W_{\text {Som }}=0.0375$ (для классической частищы $W_{\text {clas }}=0.06$ для $N=6$ ). Таким образом, раскручивание траектории для частишы Зоммерфельда сушественно запаздывает по сравнению с классическим случаем.

Отметим, что запаздывание по росту энергии приходится в основном на моменты движения внутри зазора. Это можно объяснить отличием величин ускорения частицы в электрическом поле (пропорциональном $\epsilon \approx 10^{-7}$ ) и в магнитном поле (пропорциональном $\left.v \lambda \approx 10^{-8}\right):$ как известно, поток излучения электромагнитной энергии пропорционален квадрату ускорения излучающей частицы. 


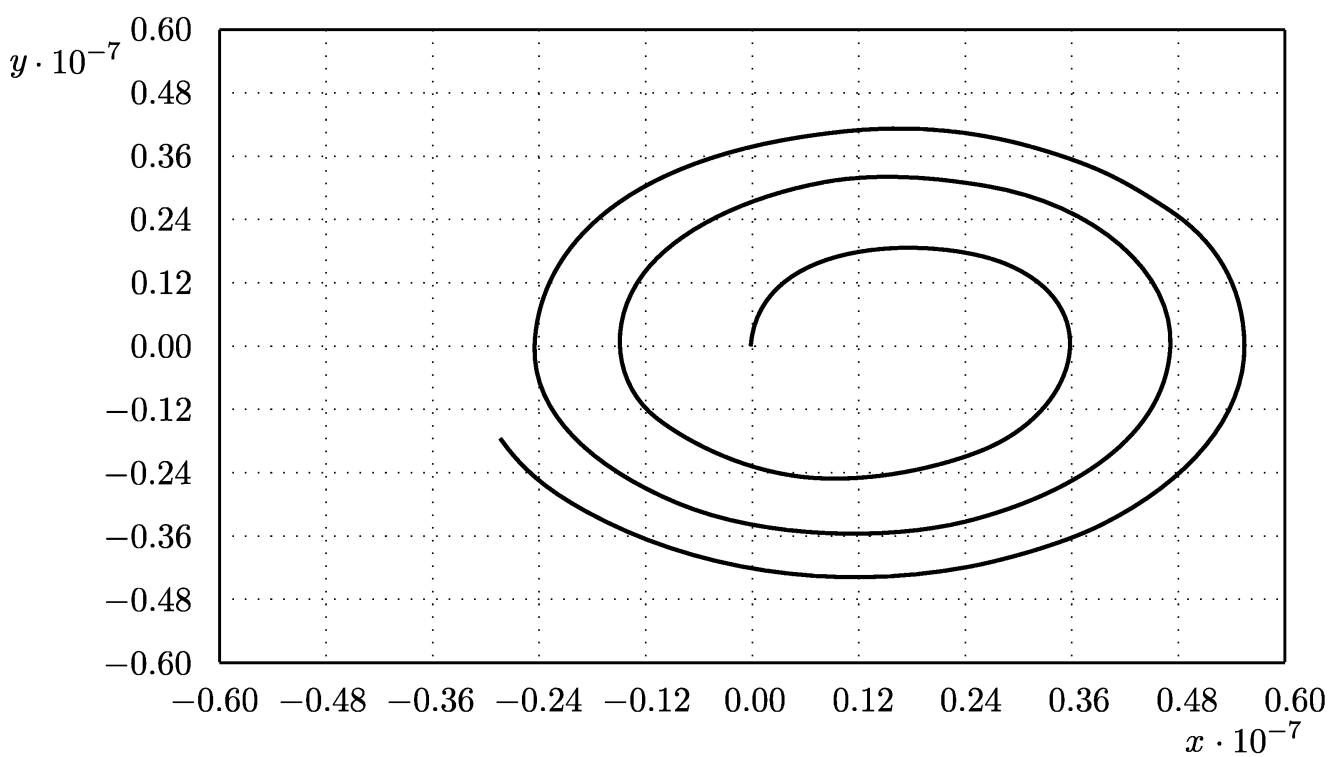

Рис. 6. Траектории частицы Зоммерфельда в циклотроне.

\section{4. РАССЕЯНИЕ РЕЗЕРФОР ДА}

Рассмотрим теперь задачу о рассеянии частицы Зоммерфельда на точечном кулоновском центре. Благодаря эффекту запаздывания такое рассеяние должно отличаться от стандартного рассеяния Резерфорда. Следует ожидать, что для частицы Зоммерфельда угол рассеяния будет меньше (отметим, что кулоновское рассеяние для точечной частицы в модели Лоренца-Дирака рассматривалось в работах [17]).

Для численного расчета воспользуемся уравнением (1).

Внешняя сила $\vec{F}_{\text {ext }}$, образованная точечным зарядом $e$ в точке $\vec{r}=0$, имеет вид

$$
\vec{F}_{\mathrm{ext}}=\int d \vec{r} \rho \frac{e \vec{r}}{r^{3}}
$$

что для сферы Зоммерфельда с плотностью заряда

$$
\rho=\frac{Q \delta(|\vec{r}-\vec{R}|-a)}{4 \pi a^{2}}
$$

дает стандартное кулоновское выражение

$$
\vec{F}_{\text {ext }}=\frac{e Q \vec{R}}{R^{3}}, \quad R>a .
$$

После введения безразмерных переменных $\vec{R}=\vec{\Pi} L, c t=\tau L$ уравнение (1) с учетом соотношения (15) примет вид

$$
\ddot{\vec{\Pi}}=k[\dot{\vec{\Pi}}(\tau-\delta)-\dot{\vec{\Pi}}(\tau)]+\lambda \frac{\vec{\Pi}}{|\vec{\Pi}|^{3}},
$$




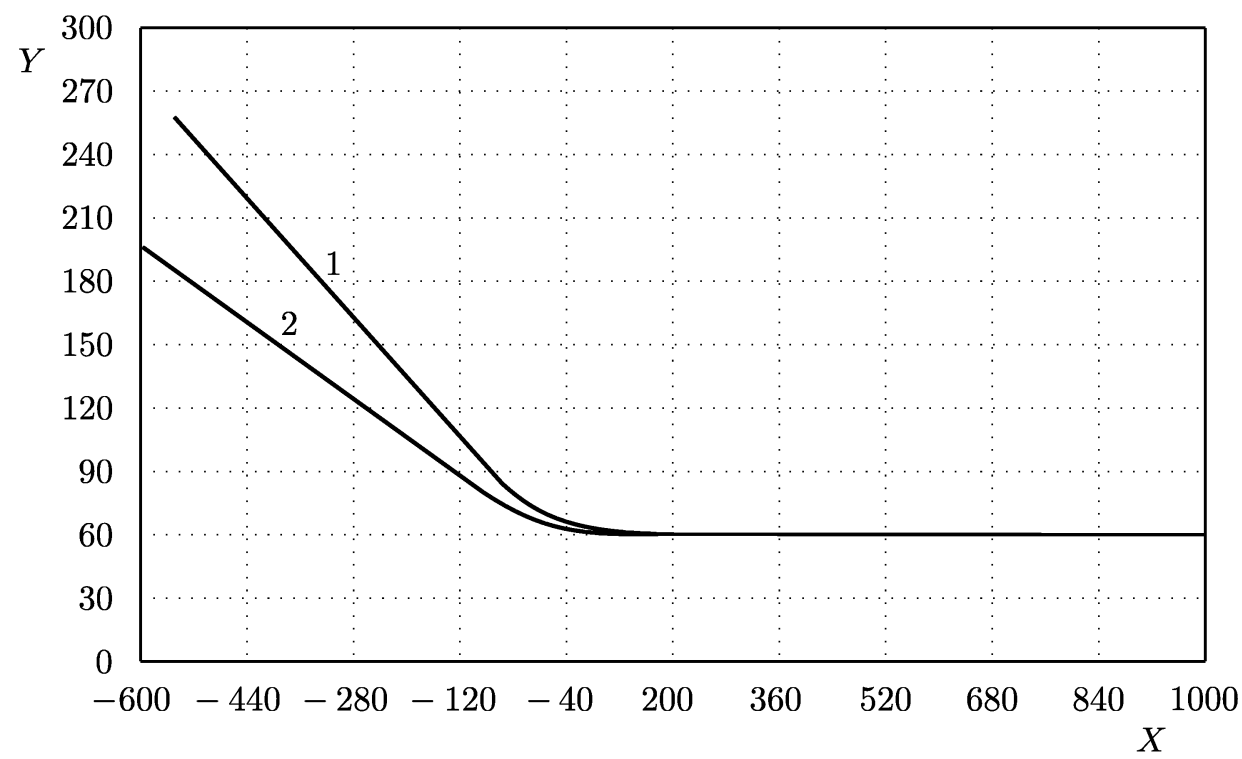

Рис. 7. Классическое рассеяние Резерфорда (кривая 1) и рассеяние в модели Зоммерфельда (кривая 2).

где

$$
k=\frac{Q^{2} L}{3 m c^{2} a^{2}}, \quad \lambda=\frac{e Q}{m c^{2} L}, \quad \delta=\frac{2 a}{L} .
$$

Если рассеяние происходит в плоскости $X Y(\vec{\Pi}=(X, Y, 0))$, то уравнение (16) записывается как

$$
\begin{aligned}
& \ddot{Y}=k[\dot{Y}(\tau-\delta)-\dot{Y}(\tau)]+\lambda Y\left(X^{2}+Y^{2}\right)^{-3 / 2}, \\
& \ddot{X}=k[\dot{X}(\tau-\delta)-\dot{X}(\tau)]+\lambda X\left(X^{2}+Y^{2}\right)^{-3 / 2} .
\end{aligned}
$$

Начальные условия при $\tau=0$ возьмем в виде

$$
X_{\text {init }}=1000, \quad Y_{\text {init }}=b
$$

( $b$ - прицельный параметр),$-\delta<\tau<0$ :

$$
\dot{X}_{\text {init }}=v_{\text {init }}, \quad \dot{Y}_{\text {init }}=0 \text {. }
$$

Выберем начальную скорость $v_{\text {init }}=-0.1$ и значения параметров

$$
k=\frac{4}{30}, \quad \lambda=0.1, \quad \delta=4.0
$$

что соответствует

$$
a=1.25 r_{\mathrm{cl}}, \quad \frac{e}{Q}=\frac{1}{16}, \quad L=\frac{5}{8} r_{\mathrm{cl}} .
$$

Численные результаты при $b=60.0$ представлены на рис. 7 , на котором видно отличие рассеяния для классической задачи Резерфорда (кривая 1 , классическое рассеяние 


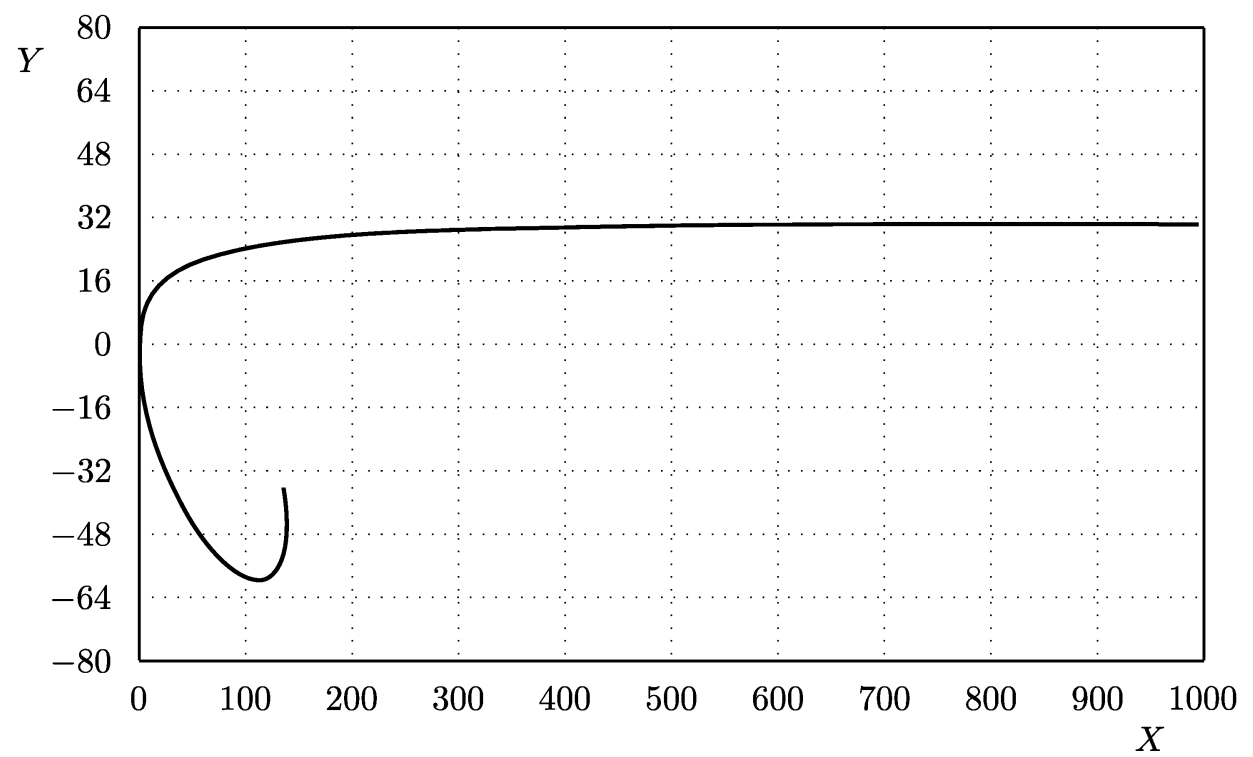

Рис. 8. Захват частицы Зоммерфельда притягиваюшим центром.

соответствует $k \equiv 0$ в (17)) от рассеяния в модели Зоммерфельда (кривая 2) (по вертикальной оси отложено $Y$, по горизонтальной - $X$ ). Таким образом, благодаря запаздыванию угол рассеяния $\theta$ для протяженной частицы меньше стандартного.

На рис. 8 видно явление захвата частицы Зоммерфельда притягиваюшим центром. Значения параметров выбраны

что соответствует

$$
\lambda=-1.0, \quad k=\frac{4}{3 \delta^{2}}, \quad \delta=5.0, \quad b=30.0,
$$

$$
L=r_{\mathrm{cl}}, \quad e=-Q, \quad a=2.5 r_{\mathrm{cl}} .
$$

Начальные условия $X_{\text {init }}=1000, Y_{\text {init }}=b,-\delta<\tau<0: \dot{X}_{\text {init }}=-0.1, \dot{Y}_{\text {init }}=0$.

В случае рассеяния Резерфорда начальные условия описывают инфинитную траекторию - из бесконечности в бесконечность. Однако для частицы Зоммерфельда траектория движения финитна и приводит к захвату: это происходит, по-видимому, из-за потерь энергии частицы на излучение.

Варьируя прицельный параметр $b$ при фиксированных значениях $\lambda=-1.0$ и $\delta=5.0$, можно вычислить критическое значение прицельного параметра, при котором возникает захват:

$$
b \leqslant b_{\text {cr }} \approx 31.40 .
$$

Заметим, что захват частищы сушествует и для модели Лоренца-Дирака, однако при других значениях прицельного параметра.

\section{5. ЗАПАЗДЫВАНИЕ ДЛЯ БРОУНОВСКОЙ ЧАСТИЦЫ}

Как уже отмечалось, уравнение движения частицы Зоммерфельда под действием внешней силы в линейном приближении можно записать в безразмерном виде как

$$
\dot{y}(\tau)+\Gamma y(\tau)=f(\tau)+k[y(\tau-\delta)-y(\tau)],
$$


где $y(\tau)$ - безразмерная скорость частицы, $\tau$ - безразмерное время, $f(\tau)$ - внешняя сила, $\Gamma$ - коэффициент вязкости окружающей среды, $\delta$ - запаздывание по времени, $k$ - такой коэффициент, что величина $k \delta$ пропорциональна отношению электромагнитной массы к механической,

$$
k \delta=\frac{Q^{2} L}{3 a^{2} m c^{2}} \frac{2 a}{L} \equiv \frac{2 r_{\mathrm{cl}}}{3} .
$$

В последнем соотношении $2 a$ - размер частицы Зоммерфельда заряда $Q$ и массы $m, L-$ масштабный множитель, $\Gamma=\Gamma_{\mathrm{cl}} L / c$, где $\Gamma_{\mathrm{cl}}-$ стандартная вязкость размерности частоты.

Если $f$ - стохастическая сила, то уравнение (18) можно рассматривать как уравнение броуновского движения заряженной частицы с временным запаздыванием. Возникает следующий вопрос: является ли эффективная температура (или, более строго, эффективная дисперсия) броуновского движения заряженной частицы такой же, как и для классической точечной незаряженной частищы?

Для ответа воспользуемся методом спектральных разложений (или методом Райса, см., например, книгу [18]). С помощью фурье-преобразования

$$
y(\tau)=\int d w e^{i w \tau} y_{w}
$$

решение основного уравнения (18) может быть представлено в виде

$$
y(\tau)=\frac{1}{2 i \pi} \int d z f(z) \int d w \frac{e^{i w(\tau-z)}}{A(w)},
$$

где $A(w)=w-i \Gamma^{\prime}+i k e^{-i w \delta}, \Gamma^{\prime}=\Gamma+k$. Тогда для стационарного процесса, т.е. для достаточно большого "времени" $\tau \gg 1 / \Gamma$, но при произвольном запаздывании $\delta$ по сравнению с $\tau$, если корреляционная функция силы $R(x, y)=\langle f(x) f(y)\rangle$ имеет вид

$$
R(x, y)=R_{0} \delta(x-y)
$$

то дисперсия

$$
D=\left\langle y(x) y^{*}(x)\right\rangle=\frac{R_{0}}{2 \pi} \int_{-\infty}^{\infty} \frac{d w}{A(w) A^{*}(w)} \equiv \frac{R_{0} \delta}{2 \pi} I
$$

где

$$
\begin{aligned}
I=I(g, G) & =\int_{-\infty}^{\infty} \frac{d z}{z^{2}+(G+g)^{2}+g^{2}-2 g(G+g) \cos z+2 g z \sin z} \equiv \\
& \equiv \int_{-\infty}^{\infty} \frac{d z}{\Phi(z ; g, G)},
\end{aligned}
$$

$g \equiv k \delta, G \equiv \Gamma \delta\left(g=2 r_{\mathrm{cl}} /(3 a), G=\Gamma_{\mathrm{cl}} 2 a / c\right)$.

Таким образом, вместо того чтобы решать исходное уравнение (18), а затем, используя найденные решения, искать дисперсию, можно сразу попытаться вычислить интеграл $(20)$. Функции $I(g, G)$ и $\Phi(z ; g, G)$ имеют следующие свойства:

1) $\Phi>0$ для $g, G>0$; 


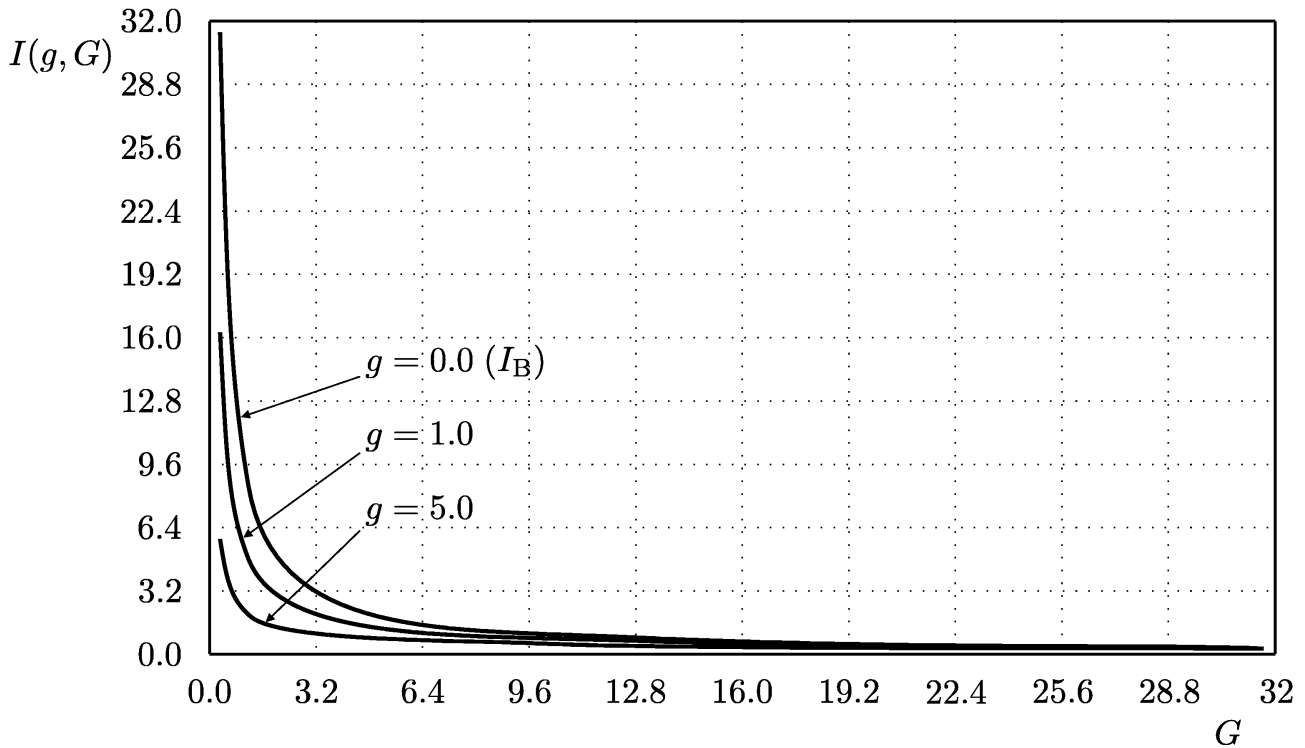

Рис. 9. Видинтеграла $I(g, G)$ из формул (19), (20) при различных значениях параметра $g$ для броуновской частицы.

2) если $g \equiv 0$ (запаздывание отсутствует), то $\Phi=z^{2}+G^{2} \equiv \Phi_{\mathrm{B}}$ и

$$
I(g \equiv 0, G)=\frac{\pi}{G} \equiv I_{\mathrm{B}}
$$

Это дает стандартный броуновский результат

$$
D=\frac{R_{0}}{2 \Gamma} \equiv D_{\mathrm{B}}
$$

3) $d \Phi / d G>0$ для всех $z, g, G$, отсюда следует, что $d I / d G<0$;

4) $I(g, G \rightarrow 0) \rightarrow \infty$, поскольку для $G \rightarrow 0$ возникает полюс $z=0$ в подынтегральном выражении $1 / \Phi: \Phi(z \rightarrow 0, g, G \rightarrow 0) \approx z^{2}(1+g)^{2}+G^{2}$, следовательно, если главный вклад в интеграл $I$ дает окрестность полюса $z=0$, то

$$
\left.I(g, G \rightarrow 0) \approx \frac{\pi}{G}\right|_{G \rightarrow 0} \frac{1}{1+g}, \quad I(g, G \rightarrow 0)<I_{\mathrm{B}}(G \rightarrow 0) .
$$

Результаты численного интегрирования функции $I$ представлены на рис. 9 и 10 . На рис. 9 для $0<G<32$ (горизонтальная ось) изображены кривые $I(g, G)$ для значений параметра $g=0.0,1.0,5.0$. На рис. 10 для $0<g<32$ (горизонтальная ось) изображены кривые $I(g, G)$ для значений параметра $G=0.1,1.0,5.0$.

Мы видим, что

$$
I(g, G)<I_{\mathrm{B}}=\frac{\pi}{G} .
$$

Таким образом, величина дисперсии для заряженной броуновской частищы (и, следовательно, величина эффективной температуры) меньше, чем для классической броуновской частишы без электрического заряда. 


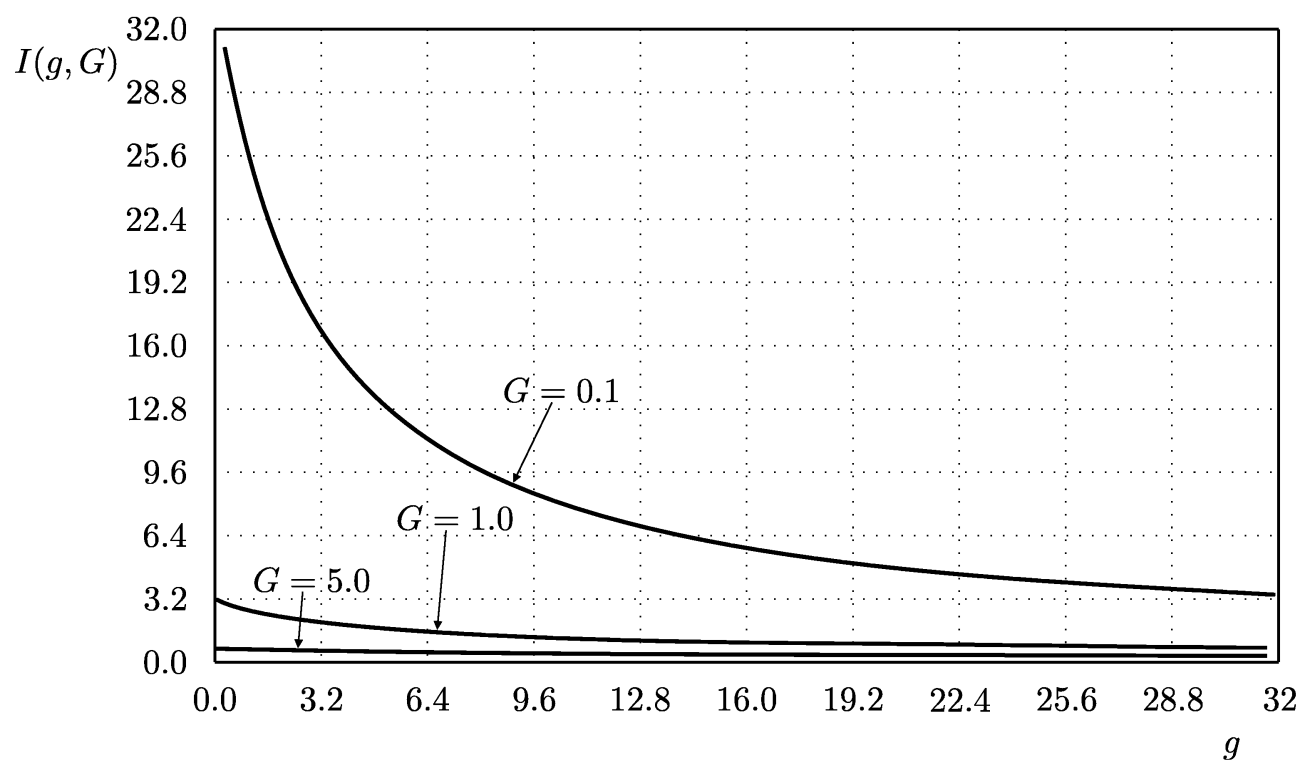

Рис. 10. Вид интеграла $I(g, G)$ из формул $(19),(20)$ при различных значениях параметра $G$ для броуновской частицы.

Если считать, что размер $а$ броуновской частицы приближенно равен $10^{-4} \mathrm{~cm}$, а масса $-10^{-17}$ г, то множитель $g$ в предыдуших формулах чрезвычайно мал, $g \approx 10^{-19}$.

\section{6. ПОТЕНЦИАЛЬНАЯ СТУПЕНЬКА}

В предыдущих примерах были получены численные результаты. Однако есть одна задача, где результат можно получить аналитически, - задача о туннелировании через потенциальную ступеньку (напомним, что эффект туннелирования нами уже рассматривался на примере плоского конденсатора в разделе 2).

Пусть частища Зоммерфельда движется в электростатическом поле $\vec{E}=\left(0,0, E_{z}\right)$, задаваемом потенциалом $\phi$ в виде ступеньки,

$$
\phi=B[\theta(z)-\theta(z-S)], \quad E_{z}=-\frac{d \phi}{d z}=-B \delta(z)+B \delta(z-S) .
$$

Тогда выражение для силы, действующей на частицу Зоммерфельда, имеет вид

$$
F_{\text {ext }}=\int d \vec{r} \rho E_{z}=\frac{Q B}{4 \pi a^{2}} \int d \vec{r} \delta(|\vec{r}-\vec{R}|-a)[-\delta(z)+\delta(z-S)] .
$$

С новыми переменными $\vec{r} \equiv \vec{\xi}+\vec{R}, d \vec{r}=d \vec{\xi}=\xi^{2} d \xi \sin \vartheta d \vartheta d \phi$, полагая $\cos \vartheta \equiv \mu$, откуда следует $z=R+\xi \mu$, интегрируя по $\phi$ и снимая дельта-функцию по $\xi$, получаем

$$
\begin{aligned}
F_{\mathrm{ext}} & =\frac{Q B}{2 a} \int_{-1}^{+1} d \mu[-\delta(R+a \mu)+\delta(R+a \mu-S)]= \\
& =\frac{Q B}{2 a}\left[-\int_{R-a}^{R+a} d w \delta(w)+\int_{R-a-S}^{R+a-S} d w \delta(w)\right] .
\end{aligned}
$$


После перехода к безразмерным величинам $y=R / L, \tau=c t / L, \delta=2 a / L$ с учетом соотношения $S=L=2 a$ уравнение (1) принимает вид

$$
\frac{d^{2} y}{d \tau^{2}}=k\left[\frac{d y(\tau-1)}{d \tau}-\frac{d y(\tau)}{d \tau}\right]+\lambda \Phi,
$$

где $k=2 Q^{2} /\left(3 m c^{2} a\right), \lambda=Q B /\left(m c^{2}\right)$ и

$$
\Phi= \begin{cases}0, & y<-1 / 2, \\ -1, & -1 / 2<y<1 / 2, \\ +1, & 1 / 2<y<3 / 2, \\ 0, & 3 / 2<y .\end{cases}
$$

Отметим, что в пределе точечности частицы

$$
\lim _{a \rightarrow 0} F_{\text {ext }}=Q B[-\delta(R)+\delta(R-S)]
$$

В этом случае получаем ньютоновское уравнение движения

$$
m \dot{v}=Q B[-\delta(R)+\delta(R-S)]=-Q B \frac{d}{d R}[-\theta(R)+\theta(R-S)] .
$$

Уравнение (24) имеет первьй интеграл

$$
m \frac{v^{2}}{2}+Q B[\theta(R)-\theta(R-S)]=\text { const }=m \frac{v_{0}^{2}}{2} .
$$

Отсюда при $0<R<S$ получаем

$$
v=\sqrt{v_{0}^{2}-\frac{2 Q B}{m}} .
$$

Найденное решение описывает прохождение барьера (ступеньки), только если начальная скорость частицы $v_{0}$ превышает критическое значение $v_{\mathrm{cr}}=\sqrt{2 Q B / m}$, или в безразмерных переменных

$$
\frac{v_{\mathrm{cr}}}{c} \equiv \dot{y}_{\mathrm{cr}}=\sqrt{2 \lambda}
$$

При $k \equiv 0$ уравнение $(23)$ имеет в области $-1 / 2<y<+1 / 2,0<\tau<t_{1}$ решение

$$
y=-\frac{1}{2}+v_{0} \tau-\frac{\lambda \tau^{2}}{2}, \quad \dot{y}=v_{0}-\lambda \tau
$$

Отсюда следует, что координата $y=+1 / 2$ достигается (при движении с $\dot{y}>0$ ) в момент времени

$$
t_{1}=\frac{v_{0}-\sqrt{v_{0}^{2}-2 \lambda}}{\lambda} .
$$

Соответственно потенциальный барьер преодолевается при $v_{0}>\sqrt{2 \lambda}$, а минимальная скорость, необходимая для преодоления барьера, есть $v_{0}=\sqrt{2 \lambda}$ (положительная часть силы при $1 / 2<y<3 / 2$ в уравнении $(23)$ не создает препятствий в движении частицы, и 
поэтому для анализа условия прохождения частицы через барьер можно рассматривать только область $-1 / 2<y<1 / 2)$. При этом $\dot{y}\left(t_{1}\right)=0$ и

$$
t_{1}=\frac{v_{0}}{\lambda}=\frac{2}{v_{0}} .
$$

Это означает, что если $t_{1}=2$, то начальная скорость должна равняться единице, а $\lambda=$ $1 / 2$, если $t_{1}=3$, то начальная скорость должна равняться $2 / 3$, а $\lambda=2 / 9$ и т.д.

Если параметр $k$ в уравнении (23) не равен нулю и если координата $y=1 / 2$ достигается в момент времени $\tau=t_{1}$ так, что $\dot{y}\left(t_{1}\right)=0$ (а значит, в предыдущие моменты времени скорость положительна), то дальнейшее движение частицы при $1 / 2<y<3 / 2$ определяется уравнением

$$
\frac{d^{2} y}{d \tau^{2}}+k \frac{d y(\tau)}{d \tau}=k \frac{d y(\tau-1)}{d \tau}+\lambda \equiv f
$$

где $f>0$. Это уравнение при условии $\dot{y}\left(t_{1}\right)=0$ имеет решение

$$
\dot{y}=e^{-k \tau} \int_{t_{1}}^{\tau} d t^{\prime} f\left(t^{\prime}\right) e^{k t^{\prime}}>0,
$$

т.е. частица барьер преодолевает и при $t>t_{1}$ продолжает свое движение.

Исходя из сказанного, приступим к построению “туннелирующих" решений уравнения (23). Предположим, что частица Зоммерфельда при своем движении достигает точку $y=-1 / 2$ при $\tau=0$, а точку $y=1 / 2-$ при $\tau=t_{1}$. Пусть при $\tau<0$ частица движется с постоянной скоростью

$$
\frac{d y}{d \tau}=v_{0}=\text { const. }
$$

Пусть выполняется условие $\dot{y}\left(t_{1}\right)=0$ (барьер преодолевается) и пусть

$$
t_{1}=2
$$

Тогда интегрирование уравнения (23) проводится на интервале $0<\tau<1,-1 / 2<y<$ $y_{1}$ и на интервале $1<\tau<2, y_{1}<y<1 / 2$.

Решение на первом интервале имеет вид

$$
\dot{y}=v_{0}-\frac{\lambda}{k}+\frac{\lambda}{k} e^{-k \tau},
$$

на втором -

$$
\begin{aligned}
\dot{y} & =v_{0}-2 \frac{\lambda}{k}+a_{0} e^{-k \tau}+\lambda \tau e^{-k \tau+k}, \\
a_{0} & =\frac{\lambda}{k}\left(e^{k}+1\right)-\lambda e^{k} .
\end{aligned}
$$

Условия $y\left(t_{1}=2\right)=1 / 2$ и $\dot{y}\left(t_{1}=2\right)=0$ сводятся к системе соотношений

$$
v_{0}=f_{1} \lambda, \quad \lambda=1 / f_{2},
$$


где

$$
\begin{aligned}
& f_{1}=\frac{2}{k}-e^{-k}\left(1+\frac{1}{k}+\frac{e^{-k}}{k}\right) \\
& f_{2}=\frac{1-e^{-k}}{k}+\frac{3-2 e^{-k}-e^{-2 k}}{k^{2}}-2 e^{-k}\left(1+\frac{1}{k}+\frac{e^{-k}}{k}\right) .
\end{aligned}
$$

Анализ функций $f_{1}(k), f_{2}(k)$ показывает, что условие $v_{0}<\sqrt{2 \lambda}$ для найденного решения выполняется в силу неравенства $f_{1} / \sqrt{2 f_{2}}<1$, т.е. происходит туннелирование (начальная скорость лежит в запрешенной области). Однако в силу соотношения $f_{1} / f_{2}>1$ начальная скорость оказьвается всегда больше единицы, $v_{0}>1$ (при этом при $k \equiv 0$ выполняется условие $v_{0}=1$ и $\lambda=1 / 2$ в соответствии с результатом (28)).

Пусть теперь вместо $t_{1}=2$ имеем

$$
t_{1}=3
$$

Тогда интегрирование уравнения (23) проводится на интервале $0<\tau<1,-1 / 2<y<$ $y_{1}$ с решением в форме (30), на интервале $1<\tau<2, y_{1}<y<y_{2}$ с решением в форме (31) и на интервале $2<\tau<3, y_{2}<y<1 / 2$ с решением в форме

$$
\begin{aligned}
\dot{y} & =v_{0}-3 \frac{\lambda}{k}+e^{-k \tau}\left[a_{1}+k e^{k}\left(a_{0}-\lambda e^{k}\right) \tau+k \lambda e^{2 k} \frac{\tau^{2}}{2}\right], \\
a_{1} & =e^{2 k}\left(\frac{\lambda}{k}-2 \lambda+2 k \lambda-\lambda e^{-k}+\frac{\lambda}{k} e^{-k}\right) .
\end{aligned}
$$

Условия $y\left(t_{1}=3\right)=1 / 2$ и $\dot{y}\left(t_{1}=3\right)=0$ сводятся к системе соотношений

$$
v_{0}=f_{3} \lambda, \quad \lambda=1 / f_{4}
$$

где

$$
\begin{aligned}
f_{3}= & \frac{e^{-3 k}}{2 k}\left[6 e^{3 k}-\left(k^{2}+2 k+2\right) e^{2 k}-(4 k+2) e^{k}-2\right], \\
f_{4}= & \frac{e^{-3 k}}{2 k^{2}}\left[(6 k+12) e^{3 k}-\left(3 k^{3}+7 k^{2}+10 k+6\right) e^{2 k}-\right. \\
& \left.-\left(12 k^{2}+10 k+4\right) e^{k}-6 k-2\right] .
\end{aligned}
$$

Анализ функций $(36),(37)$ показывает, что условие $v_{0}<\sqrt{2 \lambda}$ для найденного решения выполняется в силу неравенства $f_{3} / \sqrt{2 f_{4}}<1$, т.е. происходит туннелирование (начальная скорость лежит в запрешенной области). В отличие от предыдушего случая начальная скорость меньше единицы, но больше $2 / 3$ (при этом для $k \equiv 0$ справедливо условие $v_{0}=2 / 3$ и $\lambda=2 / 9$ в соответствии с результатом $\left.(28)\right)$.

Построение туннелирующих решений можно продолжить и дальше для $t_{1}=4$, $5,6, \ldots$ Ясно, что с ростом величины $t_{1}$ уменьшается величина минимальной скорости $v_{0}$, необходимой для преодоления барьера, но существенно усложняется форма соответствуюшего решения. 


\section{7. ЗАКЛЮЧЕНИЕ}

Мы разобрали некоторые классические задачи (кулоновское рассеяние, движение в постоянных внешних электромагнитных полях, броуновское движение) для модели Зоммерфельда протяженной частицы ("пылинки”) (некоторые эффекты были также рассмотрены в работе [6]). При этом выявлены существенные отличия результатов от классических как в количественном отношении (изменение угла при кулоновском рассеянии, уменьшение коэффициента диффузии для броуновской частищы, замедление раскрутки “пылинки" в циклотроне), так и в качественном отношении (возникновение эффекта туннелирования через потенциальный барьер). Заметим, что указанные отличия при стремлении размера частицы к нулю не исчезают. Следовательно, уравнения движения протяженного объекта не имеют гладкого предельного перехода к ньютоновским уравнениям для точки, другими словами, они неаналитичны в окрестности нулевого значения размера объекта (что можно было сразу предположить исходя из общего вида уравнения (1)).

Таким образом, теория с $a \equiv 0$ и теория с $a \neq 0$ (где $a$ - размер излучающего тела) представляют собой разные теории с принципиально разными решениями. Подобная ситуация в физике не является исключением, например, введение ненулевой массы у гравитона принципиально меняет предсказания гравитационной теории (см., например, книгу [19]).

Благодарности. Автор благодарен А. А. Логунову и С. С. Герштейну за внимание к работе и полезные замечания.

\section{Список литературы}

[1] H. Lorentz. The Theory of Electron. 2nd edition. Leipzig: Teubner, 1916; M. Abragam. Electromagnetische Theorie der Strahlung. Leipzig: Teubner, 1905.

[2] А. И. Никишов. ЖЭЭТФ. 1996. Т. 110. № 2(8). С. 511; Г. Ф. Ефремов. ЖЭТФ. 1998. T. 114. № 5(11). C. 1661

[3] F. Rohrlich. Am. J. Phys. 1997. V. 65(11). P. 1051; Phys. Rev. D. 1999. V. 60. P. 084017.

[4] F. Denef et al. Phys. Rev. E. 1997. V. 56. P. 3654.

[5] А. А. Власов. Вестн. МГУ. Сер. 3. Физика, астрономия. 1998. № 5. С. 17.

[6] А. А. Власов. Вестн. МГУ. Сер. 3. Физика, астрономия. 2001. № 6. С. 15.

[7] P. Dirac. Proc. Roy. Soc. A. 1938. V. 167. P. 148.

[8] F. Rohrlich. Classical Charged Particles. Reading, Mass.: Addison-Wesley, 1965.

[9] Д. Д. Иваненко, А. А. Соколов. Классическая теория поля. М.: ГИТТЛ, 1949.

[10] А. А. Соколов, И. М. Тернов. Релятивистский электрон. М.: Наука, 1982.

[11] Н. П. Клепиков. УФН. 1985. Т. 146. С. 317.

[12] S. Parrott. Relativistic Electrodynamics and Differential Geometry. New York: Springer, 1987.

[13] T. Erber. Fortschr. Phys. 1961. V. 9. P. 342.

[14] P. Pearle. In: Electromagnetism. Ed. D. Tepliz. New York: Plenum, 1982. P. 211.

[15] A. Yaghian. Relativistic Dynamics of a Charged Sphere. Lecture Notes in Physics. V. 11. Berlin: Springer, 1992

[16] A. Sommerfeld. Gottingen Nachrichten. 1904. V. 29; 1904. V. 363; 1905. V. 201.

[17] J. Huschilt, W. Baylis. Phys. Rev. D. 1978. V. 17. P. 985; E. Comay. J. Phys. A. 1996. V. 29. P. 2111.

[18] И. А. Квасников. Термодинамика и статистическая физика. Ч. 2. М.: МГУ, 1988

[19] A. А. Логунов. Теория гравитационного поля. М.: Наука, 2000.

Поступила в редакцию 2.VII.2001 г., после доработки 27.II.2002 г. 